\title{
CONFORMALLY FLAT HYPERSURFACES OF SYMMETRIC SPACES
}

\author{
YOSHIO MATSUYAMA \\ (Received 18 April 1983; revised 26 July 1983) \\ Communicated by K. Mackenzie
}

\begin{abstract}
In this paper we consider how much we can say about an irreducible symmetric space $M$ which admits a single hypersurface with at most two distinct principal curvatures. Then we prove that if $N$ is conformally flat, then $N$ is quasiumbilical and $M$ must be a sphere, a real projective space or the noncompact dual of a sphere or a real projective space.
\end{abstract}

1980 Mathematics subject classification (Amer. Math. Soc.): 53 C 40, 53 C 35, 53 A 30.

Recently, the following problem was proposed by B. Y. Chen and L. Verstraelen [3]: if we assume that an irreducible symmetric space $M$ admits a single submanifold with a particular property, how much can we say about the ambient space? With respect to this problem, the author showed in [4] the following: (1) If $M$ admits a (connected) locally symmetric hypersurface $N(\operatorname{dim} N \geqslant 3)$ with at most two distinct principal curvatures, then $M$ must be a sphere, a real projective space, or the noncompact dual of a sphere or a real projective space. (2) If an irreducible symmetric space $M$ admits an Einstein hypersurface $N(\operatorname{dim} N \geqslant 3)$ with at most two distinct principal curvatures, then $M$ must be of rank 1.

The purpose of this paper is to prove the following:

THEOREM. If an irreducible symmetric space $M$ admits a conformally flat hypersurfaced $N(\operatorname{dim} N \geqslant 4)$ with at most two distinct principal curvatures, then $M$ must be a sphere, a real projective space, or the noncompact dual of a sphere or a real projective space.

(C) 1985 Australian Mathematical Society $0263-6115 / 85 \$ A 2.00+0.00$ 
It is well-known that an $n$-dimensional $(n \geqslant 4)$ hypersurface $N$ in a sphere, a real projective space, or the noncompact dual of a sphere or a real projective space is conformally flat if and only if it is quasiumbilical (see [1] for instance). Hence, we know that: $A$ conformally flat hypersurface $N(\operatorname{dim} N \geqslant 4)$ with at most two distinct principal curvatures in an irreducible symmetric space is quasiumbilical ( see Theorem 8.1 of [3]).

\section{Symmetric spaces and basic formulas}

Let $M$ be a connected Riemannian symmetric space. As usual if $G$ denotes the closure of the group of isometries generated by an involutive isometry for each point of $M$, then $G$ acts transitively on $M$; hence the isotropy subgroup $H$, say at 0 , is compact and $M=G / H$. Let $\$$, $\$$ denote the Lie algebras corresponding to $G, H$, respectively. Then we call

$$
\mathfrak{S}=\mathfrak{G}+\mathfrak{M} \text {, and } \mathfrak{G}=[\mathfrak{M}, \mathfrak{M}]
$$

by the Cartan decomposition. It is well-known the space $\mathfrak{M}$ consists of the Killing vector field $X$ whose covariant derivative vanishes at 0 ; in particular, the evaluation map at 0 gives a linear isomorphism of $\mathfrak{M}$ onto $T_{0} M: X \mapsto X(0)$. Hence we have

LEMMA 1.1. For the curvature tensor $R$ at 0

$$
R(X, Y) Z=-[[X, Y], Z] \text { for } X, Y, Z \in \mathfrak{M} \text {. }
$$

LEMMa 1.2. A linear subspace $L$ of the tangent space $T_{0} M$ to a symmetric space $M$ is the tangent space to some totally geodesic submanifold $N$ of $M$ if and only if $L$ satisfies the condition $[[\mathfrak{R}, \mathfrak{R}], \mathfrak{R}] \subset \mathfrak{R}$, where

$$
\mathfrak{R}=\{X \in \mathfrak{R} ; X(0) \in L\} .
$$

Next, let $N$ be a hypersurface of an $(n+1)$-dimensional Riemannian manifold $M$. And let $\nabla$ and $\nabla^{\prime}$ be the covariant differentiations on $M$ and $N$, respectively. Then the second fundamental form $A$ of the immersion is given by

$$
\begin{gathered}
\nabla_{X} Y=\nabla_{X}^{\prime} Y+g(A X, Y) \xi, \\
\nabla_{X} \xi=-A X,
\end{gathered}
$$

for vector fields $X, Y$ tangent to $N$ and a unit vector field $\xi$ normal to $N$, where $g$ is the metric tensor of $N$ induced by the immersion from the metric tensor $g$ of $M$. The equations of Gauss and Codazzi are then given respectively

$$
\begin{aligned}
R^{\prime}(X, Y ; Z, W)= & R(X, Y ; Z, W)+g(A Y, Z) g(A X, W) \\
& -g(A X, Z) g(A Y, W),
\end{aligned}
$$




$$
R(X, Y ; Z, \xi)=g\left(\left(\nabla_{X}^{\prime} A\right) Y, Z\right)-g\left(\left(\nabla_{Y}^{\prime} A\right) X, Z\right),
$$

for vector fields $X, Y, Z, W$ tangent to $N$ and $\xi$ normal to $N$, where $R$ and $R^{\prime}$ are the curvature tensors of $M$ and $N$, respectively, and $R(X, Y ; Z, W)=$ $g(R(X, Y) Z, W)$.

The following result is basic:

Lemma 1.3 (Chen \& NagANo [2]). If an irreducible symmetric space $M$ admits a totally geodesic hypersurface, then $M$ must be a sphere, a real projective space, or the noncompact dual of a sphere or a real projective space.

\section{Proof of Theorem}

Let $N$ be a hypersurface in $M$ and $E_{1}, \ldots, E_{n}$ be an orthonormal basis of $T_{x} N$, $x \in N$. Then the Ricci tensor $S^{\prime}$ of $N$ satisfies

$$
\begin{aligned}
S^{\prime}(Y, Z) & =\sum_{i=1}^{n} R^{\prime}\left(E_{i}, Y ; Z, E_{i}\right) \\
& =S(Y, Z)-R(\xi, Y ; Z, \xi)+\operatorname{trace} A g(A Y, Z)-g\left(A^{2} Y, Z\right)
\end{aligned}
$$

for $Y, Z \in T_{x} N$, where $S$ denotes the Ricci tensor of $M$.

We suppose that there is a point $x_{0}$ at which two principal curvatures $\alpha, \beta$ are exactly distinct. Then we can choose a neighborhood $U$ of $x_{0}$ on which $\alpha \neq \beta$. We put $T_{\alpha}=\{X \in T U \mid A X=\alpha X\}$ and $T_{\beta}=\{X \in T U \mid A X=\beta X\}$. Then the equation (2.1) gives

$$
\begin{aligned}
S^{\prime}(Y, Z)= & S(Y, Z)-R(\xi, Y ; Z, \xi) \\
& +(p \alpha+(n-p) \beta) g(A Y, Z)-g\left(A^{2} Y, Z\right),
\end{aligned}
$$

where $p$ denotes the multiplicity of $\alpha$. Thus the scalar curvatures $\rho^{\prime}$ and $\rho$ of $N$ and $M$ satisfy

$$
\begin{aligned}
\rho^{\prime} & =\sum_{i=1}^{n} S^{\prime}\left(E_{i}, E_{i}\right) \\
& =\rho-2 S(\xi, \xi)+(p \alpha+(n-p) \beta)^{2}-\left(p \alpha^{2}+(n-p) \beta^{2}\right) \\
& =\frac{n-1}{n+1} \rho+p(p-1) \alpha^{2}+2 p(n-p) \alpha \beta+(n-p)(n-p-1) \beta^{2},
\end{aligned}
$$

where the last equality holds since $M$ is Einsteinian. Now, by the assumption that $N$ is conformally flat, the Weyl conformal curvature tensor of $N$ vanishes. Thus by $(2.1)^{\prime}$ and (2.2), we see that the curvature tensor $R$ of $M$ satisfies 
(2.3)

$$
\begin{aligned}
(n- & 2)\{R(X, Y ; Z, W)+g(A X, W) g(A Y, Z)-g(A X, Z) g(A Y, W)\} \\
= & g(Y, W)\left\{R(\xi, X ; Z, \zeta)-(p \alpha+(n-p) \beta) g(A X, Z)+g\left(A^{2} X, Z\right)\right\} \\
& -g(X, W)\left\{R(\xi, Y ; Z, \xi) X-(p \alpha+(n-p) \beta) g(A Y, Z)+g\left(A^{2} Y, Z\right)\right\} \\
& +g(X, Z)\left\{R(\xi, Y ; W, \xi)-(p \alpha+(n-p) \beta) g(A Y, W)+g\left(A^{2} Y, W\right)\right\} \\
& -g(Y, Z)\left\{R(\xi, X ; W, \xi)-(p \alpha+(n-p) \beta) g(A X, W)+g\left(A^{2} X, W\right)\right\} \\
& +\frac{\rho}{n+1}\{g(X, W) g(Y, Z)-g(X, Z) g(Y, W)\} \\
& -\frac{1}{n-1}\left(p(p-1) \alpha^{2}+2 p(n-p) \alpha \beta+(n-p)(n-p-1) \beta^{2}\right) \\
& \cdot\{g(X, W) g(Y, Z)-g(X, Z) g(Y, W)\}
\end{aligned}
$$

for $X, Y, Z, W$ tangent to $N$.

Let $X, Y, Z, W$ and $T$ be vector fields tangent to $N$. By differentiation of (2.3) with respect to $T$, we may obtain, after a straightforward computation, that (2.4) $(n-2)\{g(A T, X) R(W, Z ; Y, \xi)+g(A T, Y) R(Z, W ; X, \xi)$

$$
\begin{aligned}
& +g(A T, Z) R(Y, X ; W, \xi)+g(A T, W) R(X, Y ; Z, \xi) \\
& +g\left(\left(\nabla_{T}^{\prime} A\right) g(A Y, Z)-g\left(\left(\nabla_{T}^{\prime} A\right) X, Z\right) g(A Y, W)\right. \\
& \left.\quad+g(A X, W) g\left(\left(\nabla_{T}^{\prime} A\right) Y, Z\right)-g(A X, Z) g\left(\left(\nabla_{T}^{\prime} A\right) Y, W\right)\right\}
\end{aligned}
$$$$
=g(Y, W)\{-R(A T, X ; Z, \xi)
$$

$$
\begin{aligned}
& -R(\xi, X ; Z, A T)-(p T \alpha+(n-p) T \beta) g(A X, Z) \\
& \left.-(p \alpha+(n-p) \beta) g\left(\left(\nabla_{T}^{\prime} A\right) X, Z\right)+g\left(\left(\nabla_{T}^{\prime} A^{2}\right) X, Z\right)\right\}
\end{aligned}
$$$$
-g(X, W)\{-R(A T, Y ; Z, \xi)-R(\xi, Y ; Z, A T)
$$$$
-(p T \alpha+(n-p) T \beta) g(A Y, Z)
$$$$
\left.-(p \alpha+(n-p) \beta) g\left(\left(\nabla_{T}^{\prime} A\right) Y, Z\right)+g\left(\left(\nabla_{T}^{\prime} A^{2}\right) Y, Z\right)\right\}
$$

$+g(X, Z)\{-R(A T, Y ; W, \xi)$

$$
\begin{aligned}
& -R(\xi, Y ; W, A T)-(p T \alpha+(n-p) T \beta) g(A Y, W) \\
& \left.\quad-(p \alpha+(n-p) \beta) g\left(\left(\nabla_{T}^{\prime} A\right) Y, W\right)+g\left(\left(\nabla_{T}^{\prime} A^{2}\right) Y, W\right)\right\}
\end{aligned}
$$$$
-g(Y, Z)\{-R(A T, X ; W, \xi)
$$

$$
\begin{aligned}
& -R(\xi, X ; W, A T)-(p T \alpha+(n-p) T \beta) g(A X, W) \\
& \left.\quad-(p \alpha+(n-p) \beta) g\left(\left(\nabla_{T}^{\prime} A\right) X, W\right)+g\left(\left(\nabla_{T}^{\prime} A^{2}\right) X, W\right)\right\}
\end{aligned}
$$

$-\frac{1}{n-1}\left(p(p-1) T \alpha^{2}+2 p(n-p) T \alpha \beta+(n-p)(n-p-1) T \beta^{2}\right)$

$\cdot\{g(X, W) g(Y, Z)-g(X, Z) g(Y, W)\}$. 
If $X, Y, Z, W$ are vectors in $T_{\alpha}$ such that $X=W, Y=Z$ and $X, Y$ are orthonormal, then by (1.4) and (2.4) we find

$$
\begin{aligned}
&(n-2)\{2 \alpha X \alpha g(T, X)+2 \alpha X \alpha g(T, Y)+2 \alpha T \alpha\} \\
&=-2\{-2 A T \alpha+\alpha Y \alpha g(T, Y)+g\left((\alpha I-A) \nabla_{Y}^{\prime} Y, A T\right) \\
&+\alpha X \alpha g(T, X)+g\left((\alpha I-A) \nabla_{X}^{\prime} X, A T\right) \\
&\left.-(p T \alpha+(n-p) T \beta) \alpha-(p \alpha+(n-p) \beta) T \alpha+T \alpha^{2}\right\} \\
&-\frac{1}{n-1}\left(p(p-1) T \alpha^{2}+2 p(n-p) T \alpha \beta+(n-p)(n-p-1) T \beta^{2}\right) .
\end{aligned}
$$

In particular, for $X=T,(2.5)$ implies

$$
\begin{aligned}
4(n-2) \alpha X \alpha=-2\{-(2 p-1) \alpha X \alpha-(n-p) \beta X \alpha-(n-p) \alpha X \beta\} \\
-\frac{1}{n-1}\{2 p(p-1) \alpha X \alpha+2 p(n-p) \beta X \alpha \\
\quad+2 p(n-p) \alpha X \beta+2(n-p)(n-p-1) \beta X \beta\} .
\end{aligned}
$$

Let $T=X, W=\omega$ in $T_{\beta}$ and $Y, Z$ in $T_{\alpha}$ be orthonormal vectors. Then (2.4) gives

$$
-(n-2) \beta(\beta-\alpha) g\left(\nabla_{Z}^{\prime} \omega, Y\right)=0
$$

for orthonormal vectors $Y, Z$ in $T_{\alpha}$. By linearization, we find

$$
\beta_{i}\left\{g\left(\nabla_{Y}^{\prime} \omega, Y\right)-g\left(\nabla_{Z}^{\prime} \omega, Z\right)\right\}=0
$$

for orthonormal vectors $Y, Z$ in $T_{\alpha}$. Similarly, we have

$$
\begin{gathered}
\alpha g\left(\nabla_{\omega_{1}}^{\prime} X, \omega_{2}\right)=0, \\
\alpha\left\{g\left(\nabla_{\omega_{1}}^{\prime} X, \omega_{1}\right)-g\left(\nabla_{\omega_{2}}^{\prime} X, \omega_{2}\right)\right\}=0
\end{gathered}
$$

for $X$ in $T_{\alpha}$ and orthonormal vectors $\omega_{1}, \omega_{2}$ in $T_{\beta}$.

Let $Y=W, Z$ in $T_{\alpha}$ be orthonormal vectors and $T=\omega_{1}, X=\omega_{2}$ unit vectors in $T_{\beta}$. Then (2.4) gives

$$
\begin{aligned}
&(n-2)\{-\beta g\left(\omega_{1}, \omega_{2}\right) Z \alpha-\alpha(\alpha-\beta) g\left(\nabla_{\omega_{1}}^{\prime} Z, \omega_{2}\right) \\
&=\left.-\beta(\alpha-\beta) g\left(\nabla_{\omega_{1}}^{\prime} Z, \omega_{2}\right)+\beta(\alpha-\beta) g\left(\nabla_{\omega_{2}}^{\prime} Z, \omega_{1}\right)\right\} \\
&-\beta(\alpha-\beta) g\left(\nabla_{\omega_{1}}^{\prime} Z, \omega_{2}\right)+\beta g\left(\omega_{1}, \omega_{2}\right) Z \beta \\
&-(p \alpha+(n-p) \beta)(\alpha-\beta) g\left(\nabla_{\omega_{1}}^{\prime} Z, \omega_{2}\right)+\left(\alpha^{2}-\beta^{2}\right) g\left(\nabla_{\omega_{1}}^{\prime} Z, \omega_{2}\right) .
\end{aligned}
$$


For unit vectors $Y=W=\omega_{0}$ in $T_{\beta}, Z$ in $T_{\alpha}$, and $T=\omega_{1}, X=\omega_{2}$ in $T_{\beta}$ which are perpendicular to $\omega_{0}$

$$
\begin{aligned}
(n-2) & \left\{-\beta g\left(\omega_{1}, \omega_{2}\right) Z \beta+\beta(\alpha-\beta) g\left(\omega_{1}, \omega_{2}\right) g\left(\nabla_{\omega_{0}}^{\prime} Z, \omega_{0}\right)\right. \\
= & \left.-\beta(\alpha-\beta) g\left(\nabla_{\omega_{1}}^{\prime} Z, \omega_{2}\right)+\beta g\left(\omega_{1}, \omega_{2}\right) Z \beta \quad-\beta(\alpha-\beta) g\left(\nabla_{\omega_{1}}^{\prime} Z, \omega_{2}\right)\right\} \\
& -\beta(\alpha-\beta) g\left(\nabla_{\omega_{1}}^{\prime} Z, \omega_{2}\right)+\beta(\alpha-\beta) g\left(\nabla_{\omega_{2}}^{\prime} Z, \omega_{1}\right) \\
& -(p \alpha+(n-p) \beta)(\alpha-\beta) g\left(\nabla_{\omega_{1}}^{\prime} Z, \omega_{2}\right)+\left(\alpha^{2}-\beta^{2}\right) g\left(\nabla_{\omega_{1}}^{\prime} Z, \omega_{2}\right) .
\end{aligned}
$$

Subtracting (2.12) from (2.11), we obtain

$$
\begin{aligned}
\alpha\left\{(-\beta Z \alpha+\beta Z \beta) g\left(\omega_{1}, \omega_{2}\right)-\alpha(\alpha-\beta) g\left(\nabla_{\omega_{1}}^{\prime} Z, \omega_{2}\right)\right\} \\
=\alpha \beta(\alpha-\beta)\left\{g\left(\omega_{1}, \omega_{2}\right) g\left(\nabla_{\omega_{0}}^{\prime} Z, \omega_{0}\right)-g\left(\nabla_{\omega_{1}}^{\prime} Z, \omega_{2}\right)\right\}
\end{aligned}
$$

Putting $\omega_{1}=\omega_{2}$ and using (2.10), we find

$$
\alpha\left\{-\beta Z \alpha+\beta Z \beta-\alpha 1 \cdot g\left(\nabla_{\omega_{1}}^{\prime} Z, \omega_{1}\right)\right\}=0
$$

Let $X_{1}, \ldots, X_{p}, \omega_{1}, \ldots, \omega_{n-p}$ be an orthonormal basis of $T_{x} N$ such that $X_{1}, \ldots, X_{p}$ (resp. $\omega_{1}, \ldots, \omega_{n-p}$ ) forms an orthonormal basis of $T_{\alpha}$ (resp. $T_{\beta}$ ). Since $M$ is Einstein, we have

$$
\begin{aligned}
0 & =S\left(X_{i}, \xi\right) \\
& =\sum_{j=1}^{p} R\left(X_{i}, X_{j} ; X_{j}, \xi\right)+\sum_{k=1}^{n-p} R\left(X_{i}, \omega_{k} ; \omega_{k}, \xi\right) \\
& =p X_{i} \alpha+(n-p) X_{i} \beta-(n-p)(\alpha-\beta) g\left(\nabla_{\omega_{k}}^{\prime} X_{i}, \omega_{k}\right),
\end{aligned}
$$

using (2.10) for all $i, k$. From (2.13)' and (2.14) we obtain

$$
\alpha\left\{(p \alpha+(n-p) \beta) X_{i} \alpha+(n-p)(\alpha-\beta) X_{i} \beta\right\}=0 .
$$

Now, we assume that $\operatorname{dim} T_{\alpha} \geqslant 3$. Let $X, Y=Z, T=W$ be orthonormal vectors in $T_{\alpha}$. Then (2.4) gives

$$
(n-1) \alpha X \alpha=0 .
$$

If $\alpha \neq 0$, then from (2.6) we obtain $(n-p-1)(\alpha-\beta) X \beta=0$. Since we may assume $p \neq n-1$, we have $X \beta=0$. Therefore from (2.9), (2.10) and (2.13)' we obtain $g\left(\nabla_{\omega_{1}}^{\prime} Z, \omega_{2}\right)=0$ for all $\omega_{1}, \omega_{2}$ in $T_{\beta}$. If $\alpha \equiv 0$, then (2.6) gives $X \beta=0$. Then (2.11) and (2.12) imply

$$
\beta^{2}\left\{(n-p+1) g\left(\nabla_{\omega_{1}}^{\prime} Z, \omega_{2}\right)-g\left(\nabla_{\omega_{2}}^{\prime} Z, \omega_{1}\right)\right\}=0
$$


$(2.12)^{\prime}$

$$
\begin{aligned}
\beta^{2}(n-2)\left\{-g\left(\omega_{1}, \omega_{2}\right) g(\right. & \left.\left.\nabla_{\omega_{0}}^{\prime} Z, \omega_{0}\right)+g\left(\nabla_{\omega_{1}}^{\prime} Z, \omega_{1}\right)\right\} \\
& =\beta^{2}\left\{(n-p+1) g\left(\nabla_{\omega_{1}}^{\prime} Z, \omega_{2}\right)-g\left(\nabla_{\omega_{2}}^{\prime} Z, \omega_{1}\right)\right\}=0 .
\end{aligned}
$$

Putting $\omega_{1}=\omega_{2}$ in $(2.12)^{\prime}$, we have

$$
g\left(\nabla_{\omega_{0}}^{\prime} Z, \omega_{0}\right)=g\left(\nabla_{\omega_{1}}^{\prime} Z, \omega_{1}\right)
$$

for orthonormal vectors $\omega_{0}, \omega_{1}$ in $T_{\beta}$. Combining (2.14) and (2.17), we obtain $g\left(\nabla_{\omega}^{\prime} Z, \omega\right)=0$ for all $\omega$ in $T_{\beta}$. By linearization, we find

$$
g\left(\nabla_{\omega_{1}}^{\prime} Z, \omega_{2}\right)+g\left(\nabla_{\omega_{2}}^{\prime} Z, \omega_{1}\right)=0 .
$$

Summing up (2.11) $)^{\prime}$ and (2.18), we have

that is,

$$
(n-p+2) g\left(\nabla_{\omega_{1}}^{\prime} Z, \omega_{2}\right)=0,
$$

$$
g\left(\nabla_{\omega_{1}}^{\prime} Z, \omega_{2}\right)=0
$$

for all $\omega_{1}, \omega_{2}$ in $T_{\beta}$. If $\operatorname{dim} T_{\alpha}=2$, then we have only to show $X \alpha=X \beta=0$ for all unit vectors $X$ in $T_{\alpha}$, since we can make use of the above argument. Then from (2.6) and (2.15)

$$
\alpha\left\{(2 \alpha+(n-2) \beta) X_{i} \alpha+(n-2)(\alpha-\beta) X_{i} \beta\right\}=0
$$

$\left\{\left(2 n^{2}-9 n+9\right) \alpha-(n-2)(n-3) \beta\right\} X_{i} \alpha-(n-2)(n-3)(\alpha-\beta) X_{i} \beta=0$.

Hence we obtain $X_{i} \alpha=X_{i} \beta=0$. Therefore we have $R(X, Y ; Z, \xi)=0$ for all $X$, $Y, Z$ in $T U$. From Lemmas 1.1, 1.2 and 1.3 we obtain the conclusion.

\section{References}

[1] B. Y. Chen, Geometry of submanifolds (M. Dekker, New York, 1973).

[2] B. Y. Chen and T. Nagano, 'Totally geodesic submanifolds of symmetric spaces, II', Duke Math.J. 45 (1978), 405-425.

[3] B. Y. Chen and L. Verstraelen, 'Hypersurfaces of symmetric spaces', Bull. Inst. Math. Acad. Sinica 8 (1980), 201-236.

[4] Y. Matsuyama, 'Some hypersurfaces of symmetric spaces', Canad. Math. Bull. 28 (1983), 303-311.

Department of Mathematics

Chuo University

1-13-27 Kasuga, Bunkyo-ku

Tokyo 112, Japan 